\title{
Las actividades musicales preferidas de la voz de los propios niños y niñas de cuatro años. Un estudio de caso
}

Children's Favourite Musical Activities as Explained by Four-Year-Olds. A case study

\author{
Jèssica Pérez-Moreno \\ jessica.perez@uab.cat \\ Departamento de Didáctica de la Expresión Musical, Plástica y Corpora \\ Universidad Autónoma de Barcelona \\ Barcelona, España \\ ORCID: http://orcid.org/0000-0002-1685-8859
}

Laia Reverté Folch

laiarf4@gmail.com

Aula de Música

Sant Esteve Sesrovires, España

ORCID: http://orcid.org/0000-0002-4108-3338

doi: 10.7203/LEEME.43.13985

Recibido: 14-02-19 Aceptado: 23-04-19. Contacto y correspondencia: Jèssica Pérez Moreno, Departamento de Didáctica de la Expresión Musical, Plástica y Corporal, Universidad Autónoma de Barcelona, Facultad de Ciencias de la Educación. Campus UAB, 08193 Bellaterra, Barcelona. España.

\section{Resumen}

Esta investigación, que forma parte de un estudio más extenso, tiene como objetivo conocer de la voz de los propios niños y niñas cuál es su actividad musical preferida. Con esta finalidad, se selecciona una muestra de cinco niños y niñas de cuatro años de edad que asisten a la misma escuela de música y centro escolar en la provincia de Tarragona. La profesora de sensibilización musical ejerce a su vez de investigadora. Los datos para este objetivo se recogen mediante dos técnicas: la entrevista, que se realiza en casa de cada uno de los participantes y se apoya en diferentes imágenes para facilitar la expresión de los pequeños, y la narrativa visual, en la que se les pide dibujar su actividad musical preferida y compartirla con los demás. Los resultados reflejan las preferencias fluctuantes de los participantes con una clara predilección por aquellas actividades que implican hacer música con instrumentos u objetos sonoros y aquellas que conllevan una respuesta a la música mediante el juego.

Palabras clave: Educación Infantil, educación musical, actividades musicales, investigación cualitativa.

\begin{abstract}
The goal of this research, which is part of a larger study, was to find out favorite musical activities from children themselves. To this end, we selected a sample of five four-year-olds who attended the same music school and preschool in the province of Tarragona. The teacher responsible for developing musical awareness simultaneously acted as a researcher. Two methods were used to collect data: interviews, which were conducted at each of the participants' homes and supported by different images to help the children express themselves; and visual narratives, where the children were asked to draw their favorite musical activity and share it with the others. The results reflect the fluctuating preferences of the participants, but with a clear predilection for those activities that involve making music with instruments or through sound effects and those that entail a response to music through games.
\end{abstract}

Key words: Early Childhood Education, music activities, music education, qualitative research. 


\section{Introducción}

En los últimos 20 años, ha aumentado considerablemente el interés por comprender y valorar la actividad musical de los más pequeños (Young, 2016). Diversos autores han dedicado estudios a la etapa infantil (e.g. Pérez, 2011; Ilari, 2016; Tomlinson, 2013, 2015; Young, 2006a). Este interés por comprender la música de los más pequeños ha abarcado diferentes terrenos de estudio como son la identificación de actividades musicales (Campbell, 1998), las preferencias musicales (Roulston, 2006; Yim, Boo y Ebbeck, 2014) el rol del adulto en el juego de los niños (Weldemariam, 2014) o la exposición a la tecnología (McKenney y Voogt, 2010; Plowman, Stevenson, Stephen y McPake, 2012). Estas investigaciones se han desarrollado tanto en contexto familiar (Tomlinson, 2015; de Vries, 2011; Young, 2012a, 2012b; Young y Gillen, 2007) como escolar (Campbell, 1998; Gluschankof, 2005; de Vries, 2010; Pérez, 2011); demás, otros contextos formales como la escuela de música también tienen su representación (Griffin, 2009, 2010; de Vries, 2011; Young, 2012a). Finalmente, encontramos investigaciones centradas en la música en otros contextos como el transporte escolar (Campbell, 1998) u otros espacios públicos (Campbell, 1998; Custodero, Chen, Lin y Lee, 2006).

Uno de los aspectos en común de muchos de estos estudios es que en todos ellos se escucha, además de la voz de los niños, la voz de los adultos que hay a su alrededor (padres, madres, educadores...) (e.g. Young, 2012a; 2012b; Plowman, Stevenson, Stephen y McPake, 2012). La Convención de los Derechos del Niño de las Naciones Unidas establece el derecho del niño a ser escuchado y tener voz en sus contextos cotidianos (Folque, 2001). Folque (2001) también destaca la importancia de otros estudios en los que se reconoce que los niños tienen una perspectiva que puede ser distinta de la de los adultos. Además, autoras como Campbell (1998) destacan que cuando los pequeños están en su realidad cotidiana, muchas veces sin supervisión adulta, es cuando imponen sus propias reglas y cuando mejor se escuchan sus voces. Por lo tanto, escuchar sus voces permite descubrir sus identidades personales.

En esta línea, hay diversos estudios que centran su atención en la importancia de la voz de los niños para comprender sus experiencias musicales (Griffin, 2009; Ilari, 2016) y las actividades musicales que llevan a cabo (Young, 2006b; Young y Gillen, 2007). En otros casos, el motivo de escuchar la voz de los niños tiene el objetivo de mejorar la educación musical reglada (Tomlinson, 2013), tanto en la escuela (Griffin, 2009; de Vries, 2010) como en las escuelas de música (Griffin, 2010), buscando un equilibrio entre la opinión de los expertos y las necesidades de los más pequeños (Campbell, 1998; Griffin, 2009). Aun así, independientemente del contexto o de la finalidad de dichos estudios, surge en algunos autores la necesidad de trasladar a la práctica los resultados de la investigación (Young, 2016). 


\subsection{Actividad musical}

Tal y como defiende Leontiev (1959), entendemos que el niño construye y reconstruye su aprendizaje y su desarrollo por medio de la actividad práctica y cognitiva en situaciones proporcionadas por el contexto sociocultural concreto en el que vive. Las actividades musicales en las que participa están guiadas por motivos y persiguen establecer las bases de la educación musical acercando a los pequeños a los elementos de intercambio de la cultura musical a la vez que deberían aportan disfrute, alegría y bienestar (Pérez, 2011).

Siguiendo el concepto de actividad de Leontiev, para realizar una actividad hay que efectuar una serie de acciones. A pesar de que cada acción pretenda conseguir unos objetivos propios, cuando las acciones están encajadas en una actividad, lo que les da sentido son los motivos de la actividad y conseguir cumplir la finalidad de la misma. Tomando, por ejemplo, la actividad de interpretar una canción, las autoras de este trabajo proponen a los niños y niñas una serie de acciones que -según su criterio musical y partiendo de las competencias previsibles para cada franja de edad- anticipan que pueden propiciar la consecución de su finalidad. Son las experiencias previas y compartidas entre y con el grupo de niños y niñas y adulto de referencia, además de la potencialidad educativa del objeto de intercambio de las interacciones y la calidad de estas, lo que concreta y define la actividad, tal y como se propone y realiza. En palabras de Molina (1997, p.163) "las actividades educativas, como cualquier tipo de actividad, son construcciones sociales que ocurren en contextos específicos".

Los niños participan regularmente en distintos tipos de actividades musicales: cantan, bailan, escuchan, producen sonidos con diversos objetos, etc. Siguiendo a Christopher Small, se propone utilizar el término "musicking" (musicar) para referir al conjunto de estas actividades. Según define él mismo (Small, 1998, p.9) musicar es "tomar parte en cualquiera de las actividades inherentes a una función musical, ya sea interpretando, escuchando, ensayando, practicando, proporcionando el material para la función (componiendo) o bailando", por lo que cualquier forma de expresión musical queda englobada. Paralelamente la etnomusicóloga y educadora musical Patricia Campbell destaca el componente eminentemente rítmico de las actividades musicales infantiles, por lo que acuña el término rhythmicking (ritmicar) (1998) para referirse a ellas. A pesar de la evidente existencia de dichas actividades, hay pocos intentos de clasificación sistemática de las mismas. Malagarriga, Gómez y Viladot (2013), partiendo de referentes como Paynter o Schaffer, proponen la escucha, la interpretación y la creación como competencias básicas para la educación musical. Susan Kenney (2004) propone cinco rincones para la actividad musical: el de canción, el de audición, el de creación, uno para tocar instrumentos y otro para interpretar signos musicales.

Este estudio se ha basado en la propuesta de Gluschankof (2005), fundamentada en los conceptos anteriormente expuestos (musicking y rhythmicking) y que consiste en clasificar las expresiones musicales de los niños en tres tipologías: producir, responder y reproducir. Según esta autora un niño produce música, la crea, cuando está involucrado en una acción creativa 


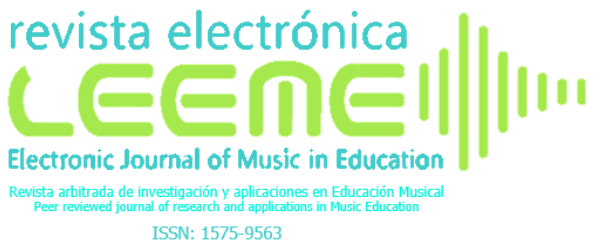

musical, como puede ser la improvisación. Esta concepción del niño creador considera que este produce música ya cuando experimenta, ya sea inventando canciones, improvisando sobre canciones conocidas o tocando un instrumento u objeto sonoro. El niño responde a la música reaccionando a ella, hecho que se puede producir de diversos modos: escuchando, bailando, uniéndose al canto o a la práctica de un instrumento y también jugando.

Indistintamente de los diferentes tipos de respuesta comentados, hay que matizar que los niños responden naturalmente a la música, expresando su comprensión de las características musicales más destacadas de la obra musical y su estilo a través del movimiento locomotor, por lo que se podría decir que el movimiento corporal es una constante en su respuesta musical. Por último, los niños también reproducen la música. La forma más común es la reproducción de una pieza creada con anterioridad por otro, y la forma más natural de hacerlo es a través del canto. Aunque el canto sea la forma más común de reproducción en estas primeras edades, también puede darse en la práctica de algún instrumento.

Cabe resaltar que el juego es una forma fundamental de aprendizaje especialmente en la etapa infantil. Buena parte de la actividad musical en esta etapa está basada en propuestas de juego. La manera de jugar de los niños ofrece a su vez informaciones sobre su desarrollo evolutivo. Fue Milderd Parten quien, en el año 1932, realizó uno de los estudios con más influencia sobre el juego social infantil, y definió tres niveles. El primero y más bajo corresponde a la actividad no social que consiste en un juego individual y solitario. A continuación, sitúa el juego en paralelo, en el que dos o más personas juegan una al lado de la otra con materiales similares, pero sin interactuar. En la cima de la clasificación encontramos el juego social interactivo, que puede tener dos vertientes: el juego asociativo y el cooperativo. Mientras que en el primer tipo cada interlocutor realiza su actividad, pero interacciona simultáneamente con el otro, en el cooperativo los participantes avanzan conjuntamente hacia la finalidad o proyecto común (Parten, 1932).

Al final de la década de los 60, Smilansky (1968) también propuso una categorización del juego, pero desde una óptica cognitiva. En la base de esta clasificación jerárquica está el juego funcional, que se basa en la repetición de actividades motrices. Un ejemplo podría ser el hecho de tirar una pelota al aire y cogerla para volver a empezar la acción. El juego constructivo viene a continuación, basado en la construcción de alguna cosa, como puede ser por ejemplo el apilar bloques de madera formando una torre. En el tercer nivel se encuentra el juego dramático, que corresponde a la representación de un hecho real, como puede ser, por ejemplo, el hacer ver que se friegan los platos en una cocina de juguete. Como tipo de juego culminante en la clasificación de Smilansky, se encuentra el juego con reglas, que consiste en seguir unas normas preestablecidas mientras se desarrolla el juego en cuestión.

\subsection{Preferencias musicales}

Conocer las preferencias musicales de los pequeños es un tema de actual interés en el ámbito de la investigación en educación musical (Yim, Boo y Ebbeck, 2014), ya que este 
conocimiento, entre otras consecuencias, puede permitir una mejora en el planteamiento de actividades musicales en contexto escolar (Griffin, 2009; Tomlinson, 2013; de Vries, 2010; Yim, Boo y Ebbeck, 2014).

Teo (2003), plantea que es más probable que los individuos dediquen más tiempo a tareas que son de su agrado, hecho que incrementa la probabilidad de repetición y el establecimiento de la preferencia. De todas formas, hay que tener presente que la accesibilidad a distintos objetos o materiales, como es el caso de los instrumentos musicales, puede determinar las preferencias de los más pequeños (Yim, Boo y Ebbeck, 2014). También es determinante el período - momento en que queramos conocer dichas preferencias. Los referentes e intereses de los niños y niñas de nuestra sociedad sufren modificaciones a la misma velocidad a la que cambia su entorno. El establecimiento de dichas preferencias necesita de un cierto nivel de desarrollo madurativo (Zenatti, 1993).

El estudio de las preferencias musicales ha sido abordado a partir de diferentes técnicas. En algunos casos se utiliza cuestionarios (Brittin, 2013; Ho, 2003; de Vries, 2010; Yim, Boo y Ebbeck, 2014) y en otros los autores abordan la temática desde una perspectiva más cualitativa partiendo de la observación y conversaciones, principalmente (e.g. Roulston, 2006; de Vries, 2011).

Debe destacarse que hay muy pocos estudios centrados en etapa de Educación Infantil, específicamente de 0 a 6 años, por lo que es necesario indagar en las preferencias musicales de esta franja de edad. Generalmente, la mayoría de investigaciones centran su interés en niños a partir de 8 años (de Vries, 2011), niños en sus primeros años de adolescencia (de Vries, 2010) o en los jóvenes (Ligero, 2009; Puente, 1996).

\section{Método}

\subsection{Objetivos}

El objetivo de esta investigación es averiguar cuál es la actividad musical preferida de los niños. Este objetivo surge de un estudio más amplio que tiene como finalidad conocer las actividades musicales que desarrollan cotidianamente los niños a partir de sus propios testimonios (Reverté, 2016).

En relación a los objetivos establecidos y a la metodología prevista, el paradigma más adecuado para situar el estudio es el interpretativo, ya que permite comprender e interpretar la realidad (Arnal, del Rincón y Latorre, 1992). Asimismo, esta investigación es exploratoria por su carácter provisional, descriptivo, así como interpretativo.

\subsection{Población y Muestra}

La muestra de este estudio está conformada por un grupo de cinco niños y niñas de cuatro años que durante el curso escolar 2015-16 cursaron Iniciación 2 en una escuela de música de la provincia de Tarragona (Tabla 1). Además, también comparten centro escolar en la misma 
población. La investigadora es la maestra de estos cinco niños y niñas en la escuela de música, de manera que la facilidad de acceso ha sido un requisito clave de cara a la selección de la muestra juntamente con el grado de familiaridad de investigadora y niños. Por tanto, el criterio de selección de la muestra ha sido el de conveniencia. Esta relación previa con las familias ha permitido que todas ellas acepten formar parte de la investigación mediante un consentimiento informado firmado por las familias y la investigadora.

Tabla 1. Datos de la muestra

\begin{tabular}{cccc} 
Pseudónimo & Fecha de nacimiento & Edad (enero 2016) & Curso escolar \\
\hline Ratolina & $16 / 03 / 2011$ & $4: 10$ & $\mathrm{P} 4$ \\
Barco Pirata & $11 / 12 / 2011$ & $4: 1$ & $\mathrm{P} 4$ \\
Dora & $30 / 10 / 2011$ & $4: 3$ & $\mathrm{P} 4$ \\
Rosie & $20 / 11 / 2011$ & $4: 2$ & $\mathrm{P} 4$ \\
Cotxe & $29 / 08 / 2011$ & $4: 5$ & $\mathrm{P} 4$
\end{tabular}

Fuente: Elaboración propia

Los nombres presentes en la tabla son ficticios ya que se pidió a cada uno de los pequeños que eligiera el suyo personalmente con tal de preservar el anonimato de los menores. Asimismo, para mostrar sus edades se ha utilizado este sistema presente en Folque (2001) en el que el primer número corresponde a los años y el segundo a los meses.

\subsection{Técnicas e instrumentos}

De acuerdo con los objetivos marcados y el planteamiento de un estudio de caso, se opta por el uso de unas técnicas de recogida de datos que permitan ponerse en contacto directo con los niños e interactuar con ellos para extraer la información de su propia voz, sin intermediarios.

La entrevista individual y la narrativa visual son las técnicas utilizadas para dar respuesta a el presente objetivo ${ }^{1}$. Para recoger los datos se utilizan los siguientes instrumentos: el diario de campo, la grabación - audio y video -, y el dibujo de los niños.

Para la estructuración de las entrevistas, se han seguido las indicaciones de Folque (2001) y se han preparado una serie de imágenes de diferentes actividades musicales, así como unos sobres con palabras referentes a distintos contextos en las que se pueden desarrollar estas actividades. Para la elaboración de este material la base ha sido el trabajo de Campbell (1998) en el que se describen distintos tipos de actividades musicales desarrollados en diferentes contextos como la casa, la escuela, la escuela de música, el transporte escolar y otros espacios públicos. Por lo tanto, en las

\footnotetext{
${ }^{1}$ En el estudio completo también se utiliza el grupo de discusión, técnica que no se va a desarrollar en este texto porque no fue necesaria para responder al objetivo aquí planteado.
}

JĖSSICA PÉREZ MORENO Y LAIA REVERTÉ FOLCH. THE CONTENT OF THIS ARTICLE IS THE SOLE RESPONSIBILITY OF THE AUTHORS. THE REVISTA ELECTRÓNICA DE LEEME AND UNIVERSITAT DE VALËNCIA ARE NOT LIABLE FOR ANY LEGAL ACTIONS THAT MAY ARISE INVOLVING THE ARTICLE'S CONTENT. REVISTA ELECTRÓNICA DE LEEME -LISTA ELECTRÓNICA EUROPEA DE MÚSICA EN LA EDUCACIÓN.HTTP://OJS.UV.ES/INDEX.PHP/LEEME/INDEX. ISSN: 1575-9563. EDITORES: UNIVERSIDAD DE VALENCIA Y JESÚS TEJADA GIMÉNEZ. (CSIC), EBSCO PREMIER, ERIH+, GALE CENGAGE LEARNING, IN-RECS, IRESIE, LATINDEX, MIAR, OCLCC, WORLDCAT, RESH, REDIB, RILM CORE JOURNALS, SUDOC, ULRICHS, ESTA REVISTA ESTÁ PUBLICADA CON EL APOYO INSTITUCIONAL DE REDIRIS-CONSEJO SUPERIOR DE INVESTIGACIONES CIENTIFICAS Y ES DE ACCESO LIBRE. CREATIVE COMMONS LICENSE 4.O BY 
imágenes utilizadas aparecen niños desarrollando actividades musicales como aparecen descritas en algunos de estos estudios: cantando, escuchando música, tocando instrumentos musicales, bailando y jugando. Todo este material es esencial para ayudar a los niños y niñas a expresarse, ya que se debe tener en cuenta que en estas entrevistas no se puede proceder de igual modo que con adultos. Según Folque (2001), la persona interesada en entrevistar niños eficientemente deberá estructurar las entrevistas de tal manera que proporcione múltiples oportunidades a los niños para que expresen lo que saben y lo que piensan.

Por lo que concierne a la segunda técnica de recogida de datos, la narrativa visual, se conceptualiza como un discurso visual creado para contar eventos (Rifà-Valls, 2011). Es por esto que se pide a los niños y niñas que dibujaran su actividad musical preferida para luego explicarla al resto de compañeros. Esta técnica permite contrastar los datos extraídos de las entrevistas. Tal y como apunta Marme Thomson (2003), en los dibujos los niños muestran sus influencias, así como sus experiencias (Rifà-Valls, 2011).

\subsection{Procedimiento de recogida de datos: la prueba piloto y las entrevistas finales}

Esta investigación está estructurada en dos fases: la fase previa y la fase principal. En la previa, se realiza la prueba piloto de la entrevista. Para ello, se escogió un único niño, de edad similar a la muestra de este estudio, con la finalidad de probar el material elaborado - formado por una serie de imágenes, tal como se ha explicado -, y de ver la respuesta del menor en una entrevista. Las condiciones en las que se desarrolló la entrevista - lugar de la casa donde se realiza y el uso de los instrumentos de recogida de datos - fueron las mismas que las del resto de entrevistas que más adelante se explicarán.

Por tanto, la entrevista piloto estuvo formada por tres partes: toma de contacto, entrevista y reflexión. La primera parte consistió en conseguir que el niño superara la vergüenza inicial y se sintiera cómodo, explicándole lo que se tenía pensado hacer y pidiéndole que pensara qué cosas hacía de música. Una vez distendido el ambiente, se pasó a la entrevista en sí. La estructura de ésta fue abierta, dejando que el pequeño explicara sus experiencias y ayudándole en caso de ser necesario con imágenes de niños realizando actividades musicales que se habían preparado previamente. Para acabar, una vez recogidos los datos necesarios, se dedicó un tiempo a la reflexión sobre la conversación. En esta última etapa proporcionó información que no había aparecido en la grabación y que se registró mediante las notas de campo.

A partir de la prueba piloto, los investigadores tomaron conciencia de la dificultad que experimentaba el entrevistado ante una entrevista abierta. Se decidió, entonces, reformular la estructura de las entrevistas para facilitar la expresión de los niños. Por este motivo, se crearon unos sobres y cartas con contextos y subcontextos (casa, escuela, escuela de música, coche, calle y otros contextos) de los que se iba a hablar, además de ampliar el número de imágenes (explicados en el apartado anterior). 
Una vez finalizada la prueba piloto, se inició la fase principal de la investigación, donde además de las entrevistas, se usó también la narrativa visual. Del mismo modo que con la prueba piloto, las entrevistas fueron grabadas en el salón de la casa de cada uno de los niños participantes. Asimismo, las entrevistas han seguido la siguiente estructura general:

- Toma de contacto. Al llegar a cada casa, se saluda a los padres y a los niños. Se prepara todo el material y se explica al pequeño en qué consiste la entrevista. Se le pide que cuente qué cosas hace de música. Asimismo, se le explica que se utilizarán algunas imágenes y sobres de colores con diversos contextos.

- Entrevista. En primer lugar, se deja que explique lo que le apetezca para facilitar la creación de un vínculo de interacción (Roulston, 2006). Según cómo avanza su discurso, se le ayuda con los sobres de contextos o las imágenes, que aparecen a partir de pequeñas pistas que dejan los niños o según el criterio de quien realiza la entrevista en ese momento. En todas las entrevistas se trata de hablar de las distintas actividades musicales que realizan en los seis contextos presentes en los sobres. Una vez tratados todos los contextos, se les formula una pregunta: "de todas las actividades de las que has hablado, ¿cuál es tu preferida?"

- Reflexión. Finalizada la entrevista, se dedica un tiempo con los padres, con los niños o bien con ambos, según la situación, para hablar sobre el contenido de la entrevista y otros aspectos.

En la mayoría de los casos, los niños estuvieron a solas con la investigadora durante el desarrollo de las entrevistas, aunque en algún caso algún progenitor prefirió estar presente. En la reflexión posterior a las entrevistas, mientras la investigadora hablaba con los padres y el niño se sentía más libre, se pudieron observar comportamientos musicales espontáneos que no habían aparecido durante la entrevista. Además, en algún caso fue posible dedicar un tiempo al juego con el pequeño, de donde también surgieron conversaciones muy ricas.

La segunda técnica de recogida de datos, la narrativa visual, se aplicó con posterioridad a las entrevistas. Para ello, se dedicó una sesión de lenguaje musical en la que los niños asistieron al aula de música. Como se ha explicado en la sección anterior, el objetivo de esta técnica era que los pequeños dibujaran individualmente su actividad musical preferida para después mostrar y explicar su dibujo a los compañeros. Como se mostrará en el siguiente apartado, la conversación entorno a el dibujo fue muy enriquecedora e indispensable para comprender el proceso que siguieron para crear su dibujo, ya que los pequeños no pudieron evitar compartir algunos aspectos mientras los realizaban.

\subsection{Análisis de los datos}

El primer paso para analizar los datos consistió en transcribir las entrevistas y las conversaciones en relación al dibujo. Para ello, se confeccionaron unas tablas que permitieron seguir 
el discurso de los niños y, a su vez, analizar los datos obtenidos. Para la categorización, se toma la propuesta de Gluschankof (2005) donde las actividades musicales se pueden clasificar en producir, responder y reproducir (ver sección 1.1. para una definición) siendo estas tres tipologías de actividades las supra categorías. La codificación se realizó manualmente mediante un código de colores sobre las tablas confeccionadas. A partir de aquí, surgieron categorías asociadas a cada una de las supra categorías según las acciones que las componían cada actividad asociada (por ejemplo: producir/tocar, responder/bailar, reproducir/cantar). A continuación, se muestra a modo de ejemplo, un fragmento de una tabla de análisis de una de las entrevistas (Tabla 2) y un fragmento de la tabla de análisis de la narrativa visual (Tabla 3).

Tabla 2. Fragmento de tabla de análisis de una entrevista

\begin{tabular}{|c|c|c|c|}
\hline Niño & $\begin{array}{l}\text { Contexto } \\
\text { (orden de } \\
\text { aparición) }\end{array}$ & Descripción & Tiempo \\
\hline \multirow[t]{10}{*}{ Dora } & Casa & Comedor: & \\
\hline & (surge & "Con el piano... cantar canciones" (lo hace sola). & $00: 17-01: 43$ \\
\hline & espontáneamente) & $\begin{array}{l}\text { "Aquí no bailo", pero en pocos segundos recapacita y dice: "Esta } \\
\text { mañana he bailado con el pito". }\end{array}$ & 02:02-02:26 \\
\hline & & No miran películas en la tele. & \\
\hline & & Tablet: "Juego a Pou", los dibujos los mira en la tele porque es & 02:27-02:44 \\
\hline & & más grande. & $05: 25-05: 40$ \\
\hline & & $\begin{array}{l}\text { Le enseño una foto con dos niñas jugando al ordenador. Ella tiene } \\
\text { una tablet y dice: "ahora ya no juego con la tablet", aunque antes } \\
\text { había dicho que sí que jugaba. } \\
\text { Cocina: }\end{array}$ & 20:20-20:34 \\
\hline & & Dice que baila en la cocina. & 03:58-04:44 \\
\hline & Calle & $\begin{array}{l}\text { Baile con los padres: "y nos cambiamos de ropa" (surge mientras } \\
\text { hablamos de que hace en casa). }\end{array}$ & 03:19-03:52 \\
\hline & & No canta por la calle, pero dice que alguna vez ha jugado a correr. & $16: 46-16: 59$ \\
\hline
\end{tabular}

Fuente: Elaboración propia

Tabla 3. Fragmento de la tabla de análisis de las conversaciones durante la narrativa visual

\begin{tabular}{|c|c|c|c|c|c|c|}
\hline $\begin{array}{c}\text { Tema de } \\
\text { conversación }\end{array}$ & Minutaje & Ratolina & $\begin{array}{l}\text { Barco } \\
\text { Pirata }\end{array}$ & Dora & Rosie & Cotxe \\
\hline \multirow[t]{2}{*}{$\begin{array}{l}\text { Actividad } \\
\text { musical que } \\
\text { dibujarán }\end{array}$} & $\begin{array}{l}00: 00- \\
08: 15\end{array}$ & & $\begin{array}{l}\text { "Yo } \\
\text { dibujo } \\
\text { un coche } \\
\text { bateria" }\end{array}$ & $\begin{array}{c}\text { "Yo mi } \\
\text { piano" } \\
\text { "Mi piano..." } \\
\text { "Pues yo el } \\
\text { niano" }\end{array}$ & $\begin{array}{c}\text { "Ah, } \\
\text { pues yo } \\
\text { el piano, } \\
\text { vale!" }\end{array}$ & $\begin{array}{c}\text { "Yo } \\
\text { también } \\
\text { una flor" }\end{array}$ \\
\hline & & & $\begin{array}{c}\text { "Yo } \\
\text { dibujo el } \\
\text { monstruo } \\
\text { de las } \\
\text { galletas" }\end{array}$ & $\begin{array}{c}\text { "Y yo voy a } \\
\text { dibujar un } \\
\text { piano! [...] y } \\
\text { ahora el } \\
\text { payaso" } \\
\text { "Yo voy a } \\
\text { dibujar un } \\
\text { piano... el } \\
\text { payaso..." }\end{array}$ & $\begin{array}{c}\text { "Un } \\
\text { piano..." } \\
\text { "Yo } \\
\text { estoy } \\
\text { pintando } \\
\text { un } \\
\text { payaso" }\end{array}$ & $\begin{array}{l}\text { "Yo una } \\
\text { galleta" }\end{array}$ \\
\hline
\end{tabular}

Fuente: Elaboración propia 
Asimismo, también se cuenta con los dibujos que realizaron los pequeños. A continuación, en la Figura 1, se muestra uno de ellos como ejemplo.

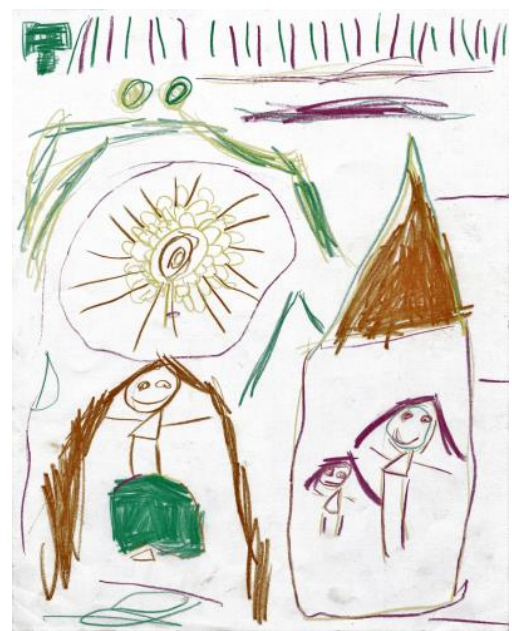

Figura 1. Dibujo de Ratolina

La información necesaria para responder al objetivo procede, como se ha visto, de dos técnicas distintas, por lo que antes de extraer los resultados fue necesario triangular ${ }^{2}$ estos datos ejecutando la siguiente tabla en la que aparecen los tipos de actividad de los que hablaron los niños. Con tal de saber en qué técnicas hablaron de cada tipo de actividad, se ha utilizado "E" para referir a la información extraída de las entrevistas y "NV" para la que surge de la narrativa visual en la Tabla 4.

Tabla 4. Contextos en los que se habla de las actividades musicales preferidas

\begin{tabular}{lccccc}
\multicolumn{1}{c}{ Tipo de actividad } & Ratolina & Barco Pirata & Dora & Rosie & Cotxe \\
\hline Producir/Tocar instrumentos & - & NV & E, NV & E, NV & NV \\
Producir/Cantar & - & - & - & - & - \\
Responder/Cantar & - & - & - & - & - \\
Responder/Escuchar & - & - & - & - & - \\
Responder/Bailar & - & - & - & - & - \\
Responder/Jugar & E & E & - & E & NV \\
Reproducir/Tocar instrumentos & - & - & - & - & - \\
Reproducir/Cantar & NV & - & - & - & E \\
\hline
\end{tabular}

Fuente: elaboración propia

\section{Discusión de resultados y conclusiones}

Antes de pasar a la discusión de los resultados propiamente dicha, se muestran los

\footnotetext{
${ }^{2}$ Se habla de triangular la información, ya que en el estudio completo se utiliza una tercera técnica de recogida de datos: el grupo de discusión. También, por qué además de la investigadora principal, el análisis es corroborado por otra investigadora. JĖSSICA PÉREZ MORENO Y LAIA REVERTÉ FOLCH. THE CONTENT OF THIS ARTICLE IS THE SOLE RESPONSIBILITY OF THE AUTHORS. THE REVISTA ELECTRÓNICA DE LEEME AND EUROPEA DE MÚSICA EN LA EDUCACIÓN.HTTP:// /OJS.UV.ES/INDEX.PHP/LEEME/INDEX. ISSN: 1575-9563. EDITORES: UNIVERSIDAD DE VALENCIA Y JESÚS TEJADA GIMÉNEZ, VISIBILIDAD DE ESTA REVISTA: SCOPUS, EMERGING SOURCES CITATION INDEX (CLARIVATE), EBSCO, CINDOC (CESIC), CITEFACTOR, COPAC, DIALNET, DICE (CSIC), DOAJ, E-REVISTAS (CSIC), EBSCO PREMIER, ERIH+, GALE CENGAGE LEARNING, IN-RECS, IRESIE, LATINDEX, MIAR, OCLC WORLDCAT, RESH, REDIB, RILM CORE JOURNALS, SUDOC, ULRICHS, ESTA REVISTA
} 
resultados expresados en la Tabla 5 y se comentan a continuación:

Tabla 5. Actividad musical preferida

\begin{tabular}{|c|c|c|c|c|}
\hline Niño & Entrevista & Tipo de actividad (E) & Narrativa visual & Tipo de actividad (NV) \\
\hline Ratolina & $\begin{array}{l}\text { Jugar al juego de } \\
\text { "L'abella zum" }\end{array}$ & $\begin{array}{l}\text { Responder/ } \\
\text { Jugar }\end{array}$ & $\begin{array}{c}\text { Cantar "L'abella } \\
\text { zum" }\end{array}$ & $\begin{array}{l}\text { Reproducir/ } \\
\text { Cantar }\end{array}$ \\
\hline $\begin{array}{l}\text { Barco } \\
\text { Pirata }\end{array}$ & $\begin{array}{c}\text { Jugar con los } \\
\text { juguetes } \\
\text { musicales }\end{array}$ & $\begin{array}{l}\text { Responder/ } \\
\text { Jugar }\end{array}$ & Tocar la batería & $\begin{array}{c}\text { Producir/ } \\
\text { Tocar instrumentos }\end{array}$ \\
\hline Dora & Tocar el piano & $\begin{array}{c}\text { Producir/ } \\
\text { Tocar instrumentos }\end{array}$ & Tocar el piano & $\begin{array}{c}\text { Producir/ } \\
\text { Tocar instrumentos }\end{array}$ \\
\hline Rosie & $\begin{array}{l}\text { Tocar el xilófono } \\
\text { Jugar a la tablet }\end{array}$ & $\begin{array}{c}\text { Producir/ } \\
\text { Tocar instrumentos } \\
\text { Responder/ } \\
\text { Jugar }\end{array}$ & Tocar el piano & $\begin{array}{c}\text { Producir/ } \\
\text { Tocar instrumentos }\end{array}$ \\
\hline Cotxe & Cantar & $\begin{array}{l}\text { Reproducir/ } \\
\text { Cantar }\end{array}$ & $\begin{array}{c}\text { Jugar con la Tablet } \\
\text { Tocar el piano }\end{array}$ & $\begin{array}{c}\text { Responder/ } \\
\text { Jugar } \\
\text { Producir/ } \\
\text { Tocar instrumentos }\end{array}$ \\
\hline
\end{tabular}

En la entrevista, Ratolina habló de responder a la música jugando, mientras que en la narrativa visual habló de reproducirla cantando. En el caso de Barco Pirata, en la entrevista habló de responder a la música jugando, mientras que en la narrativa visual habló de producirla tocando instrumentos. En ambas técnicas, Dora habló de producir música tocando instrumentos. En la entrevista, Rosie habló de dos tipos de actividad, por un lado, de producir música tocando instrumentos, y por el otro de responder jugando. En la narrativa visual, volvió a hablar de producir música tocando instrumentos. Por último, en la entrevista, Cotxe habló de un tipo de actividad en la que reproducía la música cantando, mientras que en la narrativa visual habló de dos tipos de actividad: la primera consistía en responder a la música jugando y la segunda en producirla tocando instrumentos.

Como se puede observar, la mayoría de las respuestas de los niños de este estudio difieren ante la misma pregunta o varían según el momento o situación. Este hecho se evidencia al triangular los datos. Y es que, como comenta Young (2012b) y se puede apreciar en la exposición de la narrativa visual, entrevistar los niños en parejas ayuda a la expresión de los más pequeños, aunque este tipo de acercamiento puede hacer que los niños exageren o embellezcan sus aportaciones para estar a la misma altura de las intervenciones de sus compañeros. Además, se comprueba también lo que Zenatti (1993) advertía acerca de que los niños menores de 5 años aún no tienen preferencias claras y que, a su vez, las preferencias de los individuos suelen ser transitorias (Abeles, 1980, en Zenatti, 1993). Ambas aportaciones son posibles explicaciones al hecho de que sólo dos de los niños hablaran del mismo tipo de actividad preferida en la entrevista y en la narrativa visual. Estos niños son Dora y Rosie, además ambos coincidieron en el tipo de actividad: producir tocando instrumentos. El instrumento del que nos habla Dora - el piano - se mantiene en las dos técnicas. Esta niña disponía de un piano de juguete en casa, por lo que la 


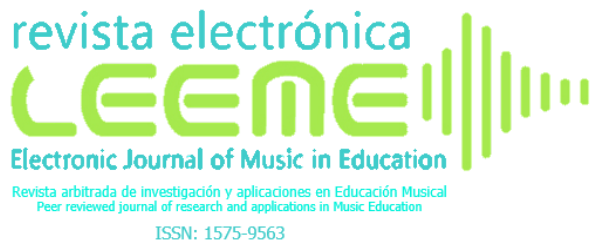

JĖsSICA PÉREZ-MORENO Y LAIA REVERTÉ FOLCH

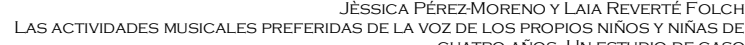
CUATRO ANROS. UN ESTUDIO DE CASO
CA DE LEEME) NÚMERO 43, PP. $19-34$ (REVISTA ELECTRÓNICA DE LEEME) NUMMERO 43, PP. 19-34 $\frac{\text { HTTPS://OJS.UV.ES/INDEX.PHP/LEEME/INDEX }}{\text { DOI: } 10.7203 / \text { LEEME } 43.13985}$

accesibilidad a este instrumento musical seguramente ha influido en su preferencia hacia él, tal y como defienden Yim, Boo y Ebbeck (2014). En el caso de Rosie, la actividad en sí a la que se refiere o el material utilizado es distinto. En cuanto a Ratolina, habla de la misma canción, "L'abella zum", aunque en la entrevista nos habla de cómo juega y en la narrativa visual sólo hace referencia a la canción en sí.

Como se ha dicho, Ratolina habló en las dos técnicas de "L'abella zum". Esta canción la aprendieron en clase de lenguaje musical y prácticamente cada semana querían jugar - de hecho, cuatro de los niños hablan en la entrevista de esta canción. Algunos autores afirman que la familiaridad con la música incrementa su preferencia hacia ella (Droe, 2006; Siebenaler, 1999 en de Vries, 2011), por las autoras de este estudio creen que este es un claro ejemplo de este fenómeno. En esta línea, una posible continuación del estudio consistiría en repetir la recogida de datos unas semanas más tarde partiendo de la hipótesis que la preferencia musical es transitoria y efímera. Sería interesante estudiar si sigue habiendo relación entre actividades realizadas en el aula y preferencias musicales de estos niños. Se quiere destacar también que, siguiendo las clasificaciones del juego social y cognitivo expuestas en este artículo, esta actividad propuesta responde a las categorías más elevadas de ambas. Cumple el requisito de ser una actividad en la que los participantes avanzan hacia un objetivo común, por lo que según Parten (1932), se trata de un juego cooperativo, y siguiendo a Smilansky (1968), se trata de un juego con reglas.

Con respecto a la naturaleza del tipo de actividad musical del que hablan los niños estudiados, se ve que de las ocho combinaciones posibles de las que hablaron en la actividad musical preferida (presentes en la Tabla 4), solamente nombran tres: producir/tocar instrumentos, responder/jugar y reproducir/cantar. De estas, las que aparecen más veces, es decir, las actividades musicales preferidas de la muestra de esta investigación son producir/tocar instrumentos y responder/jugar. En algunos estudios como el de Gluschankof (2011), el canto aparece como el comportamiento más recurrente de los niños, pero el producir tocando instrumentos y el responder jugando también son propios de estas edades, tal y cómo la misma autora define en un estudio previo (Gluschankof, 2005). En cuanto al hecho de producir música tocando instrumentos, autoras como Susan Young (2003) explican que la creación de música instrumental espontanea de los niños de tres y cuatro años consiste en poco más que un comportamiento exploratorio. Este tipo de comportamiento está especialmente ligado con el concepto de "rhythmicking" anteriormente explicado (Campbell, 1998). Por lo tanto, en este producir se entiende que los niños exploran sistemáticamente y juegan de diversas maneras, experimentando placer no solo con instrumentos musicales, sino con todo tipo de utensilios y acciones corporales (Delalande y Cornara 2010; Zenatti, 1993), involucrando gran parte de sus sentidos y exhibiendo distintos comportamientos relacionados con el juego (Gluschankof, 2011).

Los niños de este estudio han expresado también su preferencia al responder a la música jugando. Algunos de ellos nos han explicado cómo responden lúdicamente mediante movimientos corporales, enseñándonos cómo interaccionan en el juego con el resto de compañeros. A menudo usan objetos en esta actividad y mezclan comportamientos típicamente musicales con acciones

JĖSSICA PÉREZ MORENO Y LAIA REVERTÉ FOLCH. THE CONTENT OF THIS ARTICLE IS THE SOLE RESPONSIBILTY OF THE AUTHORS. THE REVISTA ELECTRÓNICA DE LEEME AND UNIVERSITAT DE VALËNCIA ARE NOT LIABLE FOR ANY LEGAL ACTIONS THAT MAY ARISE INVOLVING THE ARTICLE'S CONTENT. REVISTA ELECTRÓNICA DE LEEME - LISTA ELECTRÓNICA VISIBILIDAD DE ESTA REVISTA: SCOPUS, EMERGING SOURCES CITATION INDEX (CLARIVATE), EBSCO, CINDOC (CESIC), CITEFACTOR, COPAC, DIALNET, DICE (CSIC), DOAJ, EREVISTAS (CSIC), EBSCO PREMIER, ERIH+, GALE CENGAGE LEARNING, IN-RECS, IRESIE, LATINDEX, MIAR, OCLC WORLDCAT, RESH, REDIB, RILM CORE JOURNALS, SUDOC, ULRICHS, ESTA REVISTA ESTÁ PUBLICADA CON EL APOYO INSTITUCIONAL DE REDIRIS-CONSEJO SUPERIOR DE INVESTIGACIONES CIENTIFFICAS Y ES DEEACCESO LIBRE. CREATIVE COMMONS LICENSE 4.O BY 
de otras áreas de conocimiento. Como se sabe, el juego facilita la adquisición de habilidades como las motrices, lingüísticas, matemáticas y musicales, entre otras. Por los motivos expuestos, y además dando valor a que se trata de una actividad que los mismos niños participantes en el estudio destacan como preferida, se aboga para que las propuestas educativas musicales en la etapa de Educación Infantil contemplen el juego como base de los currículos.

En esta investigación, hemos querido recoger los datos directamente de los testimonios de los niños, fenómeno que, a pesar de que empieza a ser practicado, no es aún extendido entre quienes realizan estudios con niños de estas edades. Aventurarnos en este terreno nos ha llevado a conocer mejor las posibilidades de estos informantes y a plantearnos muy detenidamente el procedimiento de recogida de la información. De todas formas, somos conscientes de las limitaciones que conlleva esta elección y de la necesidad de ampliar la muestra del estudio para reforzar los resultados obtenidos.

\section{Referencias}

Arnal, J., del Rincón, D. y Latorre, A. (1992). Naturaleza de la investigación educativa. En Arnal, J., del Rincón, D. y Latorre, A. (Ed.), Investigación educativa: fundamentos y metodología (pp.24-49). Barcelona: Labor.

Brittin, R. V. (2013). Young Listeners' Music Style Preferences: Patterns Related to Cultural Identification and Language Use. Journal of Research in Music Education, 61 (4), 415-430. doi: $10.1177 / 0022429413509108$

Campbell, P. S. (1998). Songs in their heads: music and its meaning in children's lives. New York: Oxford University Press.

Custodero, L. A., Chen, J. J., Lin, Y. C. y Lee, K. (2006). One day in Taipei: in touch with children's spontaneous music making. En L. Suthers (Ed.), Touched by Musical Discovery Disciplinary and Cultural Perspectives: Proceedings of the ISME Early Childhood Music Education Commission Seminar. Chinese Cultural University, Taipei, Taiwan (pp.84-91). Sidney: Macquarie University.

Delalande, F. y Cornara, S. (2010). Sound explorations from the ages of 10 to 37 months: the ontogenesis of musical conducts. Music Education Research, 12 (3), 257-268. doi: $10.1080 / 14613808.2010 .504812$

Droe, K. (2006). Music preference and music education: A review of the literature. Update: Applications of Research in Music Education, 24, 23-32. doi: 10.1177/87551233060240020103

Folque, M.A. (2001). Interviewing young children. En G. Mac Naughton, S. A. Rolfe e I. SirajBlatchford (Eds.) (2010), Doing Early Childhood Research: International perspectives on 
theory \& practice (pp.239-260). Berkshire, England: Open University Press, McGraw-Hill Education.

Gluschankof, C. (2005). Spontaneous musical behaviors in Israeli Jewish and Arab kindergartens -Searching for universal principles within cultural differences (Tesis inédita de Doctorado). Jerusalem, Hebrew University of Jerusalem.

Gluschankof, C. (2011). Reflecting on the concept: "musical behaviors of young children". Proceedings del European Network Music Educators and Researchers of Young Children Conference (pp. 1-12). Helsinki Metropolia University. Helsinki, Finland. Disponible en: https://www.academia.edu/5950250/Reflecting on the concept musical behaviors of young children

Griffin, S. M. (2009). Listening to children's music perspectives: In-and-out-of-school thoughts. Research Studies in Music Education, 31, 161-177. doi: 10.1177/1321103X09344383

Griffin, S. M. (2010). Inquiring Into Children's Music Experiences: Groundings in Literature. Applications of Research in Music Education, 28 (2), 42-49. doi: 10.1177/8755123310361764

Ho, W. (2003). Gender Differences in Instrumental Learning, Preferences for Musical Activities and Musical Genres: A Comparative Study on Hong Kong, Shanghai and Taipei. Research Studies in Music Education, 20 (1), 60-76. doi: 10.1177/1321103X030200010401

Ilari, B. (2016). Music in the early years: Pathways into the social world. Research Studies in Music Education, 38 (1), 23-39. doi: 10.1177/1321103X16642631

Kenney, S. (2004). The importance of music centers in the early childhood class. General Music Today, 18 (1) 28-36. doi: 10.1177/10483713040180010106

Leontiev, A. (1959). El desarrollo del psiquismo. Madrid: Akal.

Ligero, A. (2009). Las preferencias musicales de los jóvenes en el aula. Eufonía, 46, 7-15.

Malagarriga, T., Gómez, I. y Viladot, L. (2013). ¿Cómo formar personas competentes? En T. Malagarriga y M. Martínez (Eds.), Todo se puede expresar con música. Los niños y niñas de 4 a 7 años piensan la música, hablan de música, crean música (pp.19-32). Barcelona: Dinsic, Publicacions Musicals.

Marme Thompson, C. (2003). Kinderculture in the art classroom: Early childhood art and the mediation of culture. Studies in Art Education, 44 (2), 135-146. doi: 10.1080/00393541.2003.11651734

Mckenney, S. y Voogt, J. (2010). Technology and young children: How 4-7 year olds perceive their own use of computers. Computers in Human Behavior, 26, 656-664. doi: 10.1016/j.chb.2010.01.002

JĖSSICA PÉREZ MORENO Y LAIA REVERTÉ FOLCH. THE CONTENT OF THIS ARTICLE IS THE SOLE RESPONSIBILITY OF THE AUTHORS. THE REVISTA ELECTRÓNICA DE LEEME AND UNIVERSITAT DE VALĖNCIA ARE NOT LIABLE FOR ANY LEGAL ACTIONS THAT MAY ARISE INVOLVING THE ARTICLE'S CONTENT. REVISTA ELECTRÓNICA DE LEEME -LISTA ELECTRÓNICA VISIBILIDAD DE ESTA REVISTA: SCOPUS, EMERGING SOURCES CITATION INDEX (CLARIVATE), EBSCO, CINDOC (CESIC), CITEFACTOR, COPAC, DIALNET, DICE (CSIC), DOAJ, E-REVISTAS (CSIC), EBSCO PREMIER, ERIH+, GALE CENGAGE LEARNING, IN-RECS, IRESIE, LATINDEX, MIAR, OCLC WORLDCAT, RESH, REDIB, RILM CORE JOURNALS, SUDOC, ULRICHS, ESTA REVISTA
ESTA PUBLICADA CON EL APOYO INSTITUCIONAL DE REDIRIS-CONSEJO SUPERIOR DE INVESTIGACIONES CIENTIFICAS Y ES DE ACCESO LIBRE. CREATIVE COMMONS LICENSE 4.0 BY 
Molina, L. (1997). Participar en contextos de aprendizaje y desarrollo. Bases psicopedagógicas para proyectar y compartir situaciones educativas. Barcelona: Paidós.

Parten, M. B. (1932). Social participation among pre-school children. Journal of Abnormal and Social Psychology, 27, 243-269.

Pérez, J. (2011). La música a la vida quotidiana d’infants de dos anys. Anàlisi de situacions musicals que es desenvolupen en context escolar. Tesis doctoral. Bellaterra: Universidad Autónoma de Barcelona. Recuperado de: http://www.tdx.cat/handle/10803/96096

Plowman, L., Stevenson, O., Stephen, C. y McPake, J. (2012). Preschool children's learning with technology at home. Computers \& Education, 59, 30-37. doi: 10.1016/j.compedu.2011.11.014

Puente, E. (1996). Preferencias musicales de los jóvenes fuera de las aulas. Eufonía, 5, 81-94.

Reverté, L. (2016). Estudi de les activitats musicals quotidianes dels infants de 5 anys. Trabajo final de máster no publicado. Bellaterra: Universidad Autònoma de Barcelona.

Rifà-Valls, M. (2011). Experimenting with Visual Storytelling in Students' Portfolios: Narratives of Visual Pedagogy for Pre- Service Teacher Education. International Journal of Art and Design Education, 30 (2), 293-306. doi: 10.1111/j.1476-8070.2011.01674.x

Roulston, K. (2006). Qualitative Investigation of Young Children's Music Preferences. International Journal of Education \& the Art, 7 (9), 1-23. Recuperado de: http://www.ijea.org/v7n9/

Small, C. (1998). Musicking: The Meanings of Performing and Listening. Hanover, N. H.: Wesleyan University Press.

Smilansky, S. (1968). The effects of socio-dramatic play on disadvantaged preschool children. New York: Wiley

Teo, T. (2003). Relationship of selected musical characteristics and music preference. Visions of Research in Music Education, 3, 1-20 Recuperado de: http://wwwusr.rider.edu/ vrme/v3n1/visions/teorelationship $\% 20$ of $\% 20$ selected $\% 20$ musical $\% 20$ characteristics.pdf

Tomlinson, M. M. (2013). Literacy and Music in Early Childhood: Multimodal Learning and Design. SAGE Open, (3), 1-10. doi: 10.1177/2158244013502498

Tomlinson, M. M. (2015). Young Children's Music Play Ideas: Two Case Studies of Syncretic Literacy Practice in Classroom and Home Settings. International Journal of Early Childhood, 47 (1), 119-134. doi: 10.1007/s13158-014-0128-3 123

JĖSSICA PÉREZ MORENO Y LAIA REVERTÉ FOLCH. THE CONTENT OF THIS ARTICLE IS THE SOLE RESPONSIBILITY OF THE AUTHORS. THE REVISTA ELECTRÓNICA DE LEEME AND UNIVERSITAT DE VALËNCIA ARE NOT LIABLE FOR ANY LEGAL ACTIONS THAT MAY ARISE INVOLVING THE ARTICLE'S CONTENT. REVISTA ELECTRÓNICA DE LEEME -LISTA ELECTRÓNICA EUROPEA DE MÚSICA EN LA EDUCACIÓN-HTTP://OJS.UV.ES/INDEX.PHP/LEEME/INDEX. ISSN: 1575-9563. EDITORES: UNIVERSIDAD DE VALENCIA Y JESÚS TEJADA GIMENNEZ. (CSIC), EBSCO PREMIER, ERIH+, GALE CENGAGE LEARNING, IN-RECS, IRESIE, LATINDEX, MIAR, OCLC WORLDCAT, RES, REDIB, RILM CORE JOURNALS, SUDOC, ULRICHS, ESTA REVISTA
ESTÁ PUBLICADA CON EL APOYO INSTITUCIONAL DE REDIRIS-CONSEJO SUPERIOR DE INVESTIGACIONES CIENTIFICAS Y ES DE ACCESO LIBRE. CREATIVE COMMONS LICENSE 4.O BY 
de Vries, P. (2010). What we want: the music preferences of upper primary school students and the ways they engage with music. Australian Journal of Music Education, 1, 3-16.

de Vries, P. (2011). An 8-year-old's engagement with preferred music: A case study. Research Studies in Music Education, 33 (2), 161-177. doi: 10.1177/1321103X11424195

Weldemariam, K. T. (2014). Cautionary Tales on Interrupting Children's Play: A Study from Sweden. Childhood education, 90 (4), 265-271. doi: 10.1080/00094056.2014.935692

Yim, H. Y. B.; Boo, Y. L. y Ebbeck, M. (2014). A Study of Children's Musical Preference: A Data Mining Approach. Australian Journal of Teacher Education, 39 (2), 21-34. doi: 10.14221/ajte.2014v39n2.5

Young, S. (2003). Time-space structuring in spontaneous play on educational percussion instruments among three- and four-year-olds. British Journal of Music Education, 20 (1), 45-59. doi: $10.1017 / \mathrm{S} 0265051702005284$

Young, S. (2006a). Interactive Music Technologies in Early Childhood Music Education. 9th International Conference on Music Perception and Cognition. Alma Mater Studiorum University of Bologna, (pp.1207-1211). Bolonia.

Young, S. (2006b). Seen but not heard: Young children's improvised singing and Educational Practice. Contemporary Issues in Early Childhood, 7 (3), 270-280. doi: 10.2304/ciec.2006.7.3.270

Young, S. (2012a). MyPlace, MyMusic: An International Study of Musical Experiences in the Home among Seven-year-olds. Israel Studies in Musicology Online, 10, 1-15.

Young, S. (2012b). Theorizing musical childhoods with illustrations from a study of girls's karaoke use at home. Research Studies in Music Education, 34 (2), 113-127. doi: $10.1177 / 1321103 \mathrm{X} 12466137$

Young, S. (2016). Early childhood music education research: An overview. Research Studies in Music Education, 38 (1), 9-21. doi: 10.1177/1321103X16640106

Young, S. y Gillen, J. (2007). Toward a Revised Understanding of Young Children's Musical Activities: Reflections from the "Day in the Life" Project. Current Musicology, 84, 79-99. doi: 10.7916/D81N7ZR0

Zenatti, A. (1993). Children's musical cognition and taste. En T. J. Tighe y W. J. Dowling (Eds.), Psychology and music: The understanding of melody and rhythm (pp.177-196). Hillsdale, NJ: Lawrence Erlbaum Associates: Hillsdale, NJ. 